\title{
Silicon strip detectors for two-dimensional soft X-ray imaging at normal incidence
}

\author{
P. Rato Mendes ${ }^{\mathrm{a}, *}$, M.C. Abreu ${ }^{\mathrm{a}}$, G. Baldazzi ${ }^{\mathrm{b}}$, D. Bollini ${ }^{\mathrm{b}}$, A.E. Cabal \\ Rodriguez ${ }^{c}$, W. Dabrowski ${ }^{\mathrm{d}}$, A. Diaz Garcia ${ }^{\mathrm{c}}$, M. Gambaccini ${ }^{\mathrm{e}}$, P. Giubellino, \\ M. Gombia ${ }^{\mathrm{b}}$, P. Grybos ${ }^{\mathrm{d}}$, M. Idzik ${ }^{\mathrm{d}, \mathrm{f}}$, A. Marzari-Chiesa ${ }^{\mathrm{g}}$, L.M. Montano ${ }^{\mathrm{h}}$,

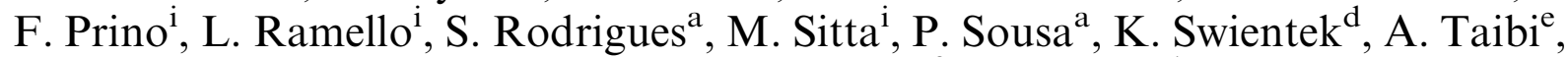 \\ A. Tuffanelli, R. Wheadon ${ }^{\mathrm{f}}$, P. Wiacek ${ }^{\mathrm{d}}$ \\ ${ }^{a}$ LIP and University of Algarve, Av. Elias Garcia 14/1, Lisboa 1000-149, Portugal \\ ${ }^{\mathrm{b}}$ Dipartimento di Fisica dell'Università di Bologna and INFN, Bologna, Italy \\ ${ }^{\mathrm{c}}$ CEADEN, Havana, Cuba \\ ${ }^{\mathrm{d}}$ Faculty of Physics and Nuclear Techniques, Academy of Mining and Metallurgy, Cracow, Poland \\ ${ }^{\mathrm{e}}$ Dipartimento di Fisica dell'Università di Ferrara and INFN, Ferrara, Italy \\ ${ }^{\mathrm{f}}$ INFN, Torino, Italy \\ ${ }^{\mathrm{g}}$ Dipartimento di Fisica Sperimentale dell'Università di Torino and INFN, Torino, Italy \\ ${ }^{\mathrm{h}}$ CINESTAV, Mexico City, Mexico

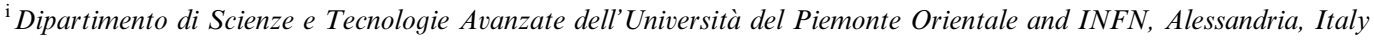

\begin{abstract}
A simple prototype system for static two-dimensional soft X-ray imaging using silicon microstrip detectors irradiated at normal incidence is presented. Radiation sensors consist of single-sided silicon detectors made from $300 \mu \mathrm{m}$ thick wafers, read by RX64 ASICs. Data acquisition and control is performed by a Windows PC workstation running dedicated LabVIEW routines, connected to the sensors through a PCI-DIO-96 interface.

Two-dimensional images are obtained by scanning a lead collimator with a thin slit perpendicular to the strip axis, along the whole detector size; the several strip profiles (slices) taken at each position are then put together to form a planar image.

Preliminary results are presented, illustrating the high-resolution imaging capabilities of the system with soft X-rays. (C) 2003 Elsevier B.V. All rights reserved.
\end{abstract}

PACS: 29.40.Wk; 87.59.Hp

Keywords: Soft X-ray imaging; Silicon microstrip detectors; RX64 readout chip

*Corresponding author. Tel.: + 351-21-797-3880; fax: + 35121-793-4631.

E-mail address: rato@lip.pt (P. Rato Mendes).

\section{Introduction}

Medical X-ray imaging has seen considerable improvements in recent years with the advent of digital radiology, based on direct detection systems 
such as flat-panel detectors, or indirect systems such as computed radiology image plates [1]. These new systems use solid-state detectors instead of film as radiation sensors, and perform better than standard film-cassette systems in terms of image contrast and resolution, with all the advantages of digital imaging processing and storage, and an additional reduction of dose delivered to the patient [2].

In this paper, we present a simple prototype system for two-dimensional digital X-ray imaging using the silicon microstrip detectors and VLSI readout electronics developed for high-resolution dual-energy mammography and angiography, as presented by Ramello et al. [3], but with X-rays hitting the silicon detectors perpendicularly. With low energy photons (less than $10 \mathrm{keV}$ ) a sufficient photoelectric conversion efficiency can be attained with thin detectors, and the simple geometry and readout of the system yields good imaging results.

\section{Experimental set-up and method}

The experimental set-up used in this work is represented schematically in Fig. 1. Silicon micro- strip detectors made from $300 \mu \mathrm{m}$ thick wafers by ITC-IRST (Trento, Italy) are used as radiation sensors, each with 132 strips at $100 \mu \mathrm{m}$ pitch and a strip length of $10.0 \mathrm{~mm}$. The charge from each strip is collected by an RX64 ASIC, a full-custom chip specifically designed for the readout of position-sensitive silicon X-ray detectors [4], with 64 channels each featuring a low noise preamplifier followed by a shaper, a discriminator and a 20-bit pseudorandom counter. Chip control and addressing is made through an $\mathrm{I} / \mathrm{O}$ bus, and the chip can also be calibrated externally.

Silicon detectors and RX64 chips are ACcoupled, mounted on a full-custom printed circuit board (PCB), connected by wire bonds. The chip is biased with 3.0, 4.0 and $4.5 \mathrm{~V}$, while the detector operates at overdepletion with $70 \mathrm{~V}$ reverse bias. Two PCB versions were used in this work: one with a single chip covering 64 channels and an active area of $6.4 \times 10 \mathrm{~mm}^{2}$, the other with two chips featuring twice the channels and area.

The data acquisition system consists of a PC workstation running LabVIEW 6i software from National Instruments. A PCI-DIO-96 interface is used to communicate with the RX64 chips, addressing, resetting, starting and stopping

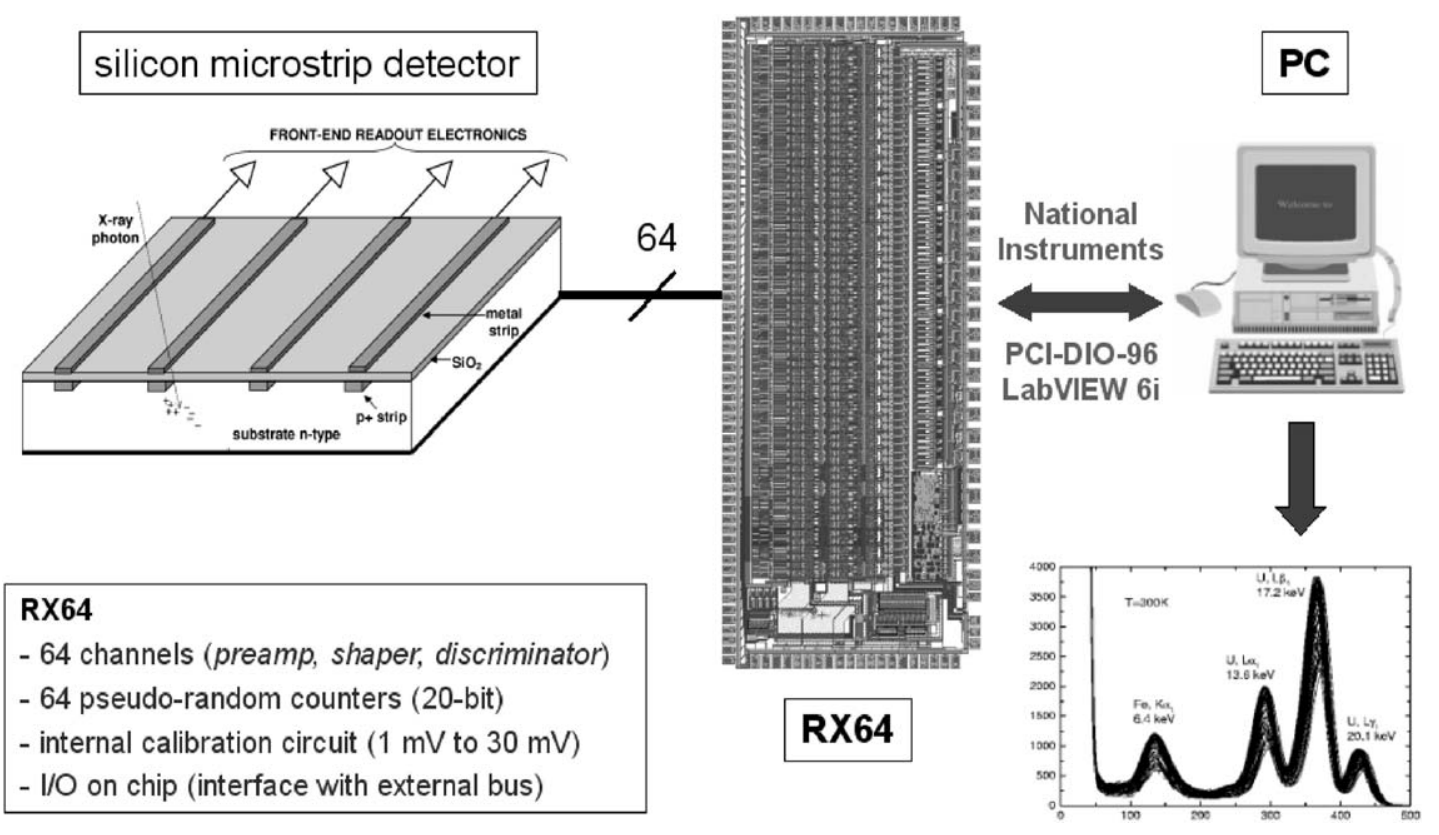

Fig. 1. Block diagram of the prototype detection system used in this work (see text for details). 
counters, and retrieving their contents. Data are presented as counts per channel for a given threshold value, or as counts per threshold for a given channel.

Two-dimensional images are obtained by scanning a thin slit on a lead collimator across the microstrip detectors under irradiation. The collimator is actuated with a stepper motor synchronized with the data acquisition system through the PCI-DIO-96 interface, so that for each slit position an image profile is acquired; the final image is obtained via software by juxtaposing all the different profiles. A photograph of the scanning device mounted over a detector board is presented in Fig. 2a; a detail of the collimator geometry is shown schematically in Fig. $2 b$.

The prototype has been calibrated and the detection efficiency has been evaluated before imaging tests were made.

Energy calibration involved several radioactive sources, both directly and through X-ray fluorescence of selected materials. An integral energy spectrum is acquired by scanning the digital threshold value and summing the counts of all strips at each discriminator setting for a given time (Fig. 3a); smoothing and differentiation of this gives a differential energy spectrum, whose peaks are then fitted with gaussian-shaped distributions

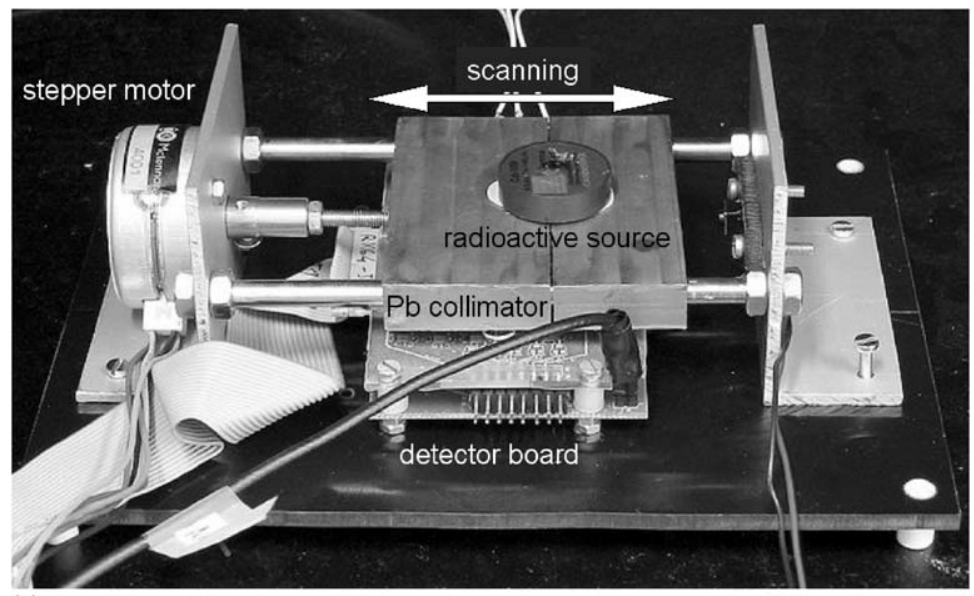

(a)

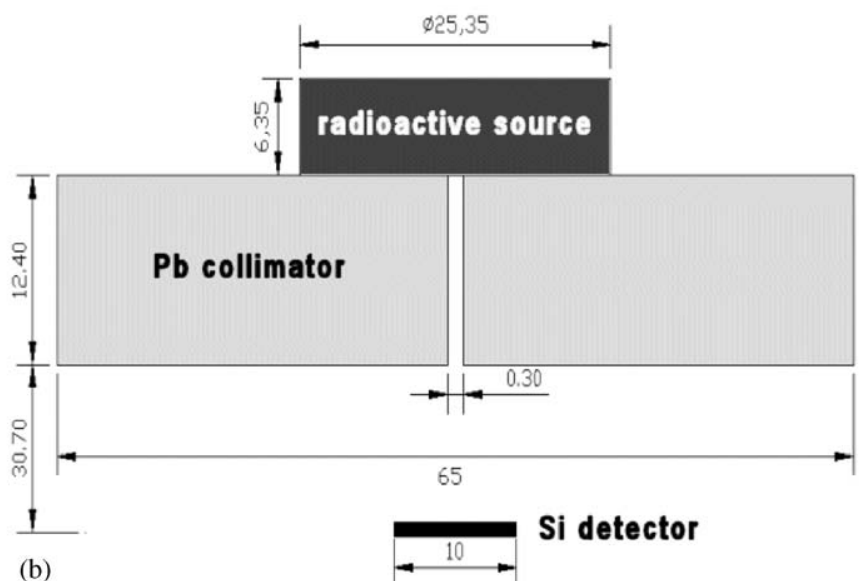

Fig. 2. Scanning system used in this work with labels indicating the main components: (a) general view; and (b) detail of the collimator geometry (dimensions in $\mathrm{mm}$ ). 

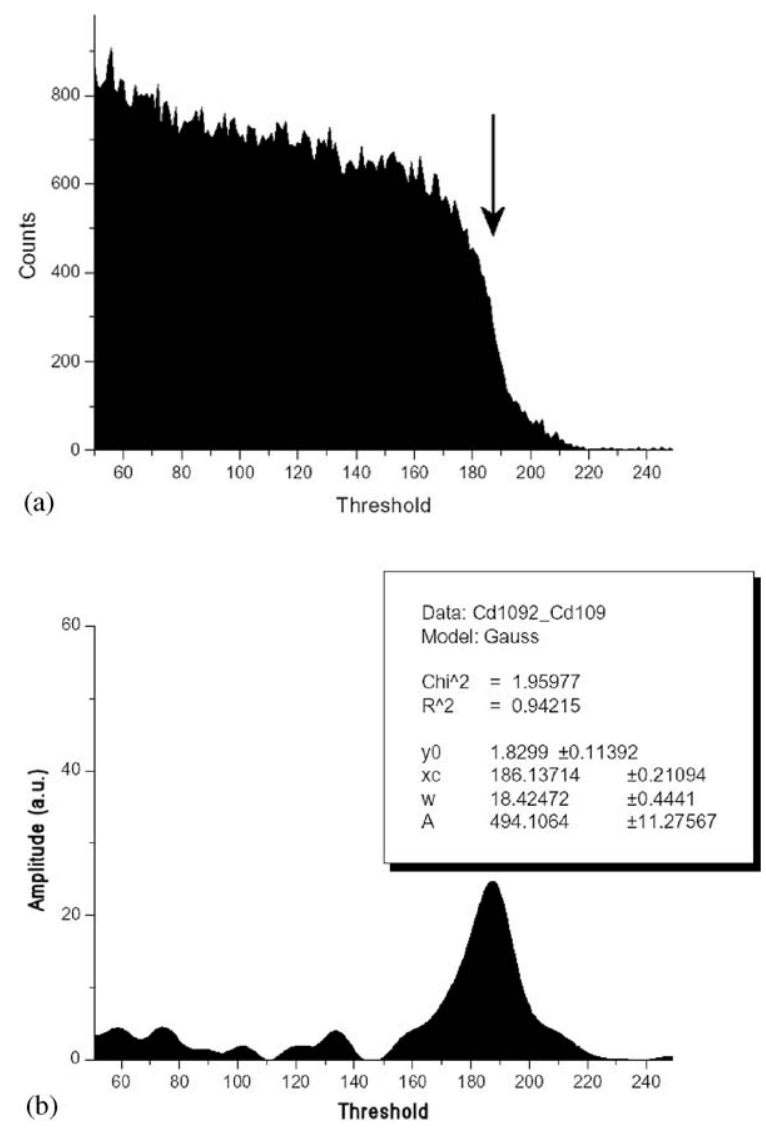

Fig. 3. (a) Integral spectrum obtained with a ${ }^{109} \mathrm{Cd}$ radioactive source (counts summed over 64 strips); and (b) the same spectrum after smoothing and differentiation (Ag K X-rays corresponding to a threshold value close to 186 , indicated by an arrow).

in order to find the correspondence between threshold value and energy (Fig. 3b). The comprehensive data from all sources was used to linearly fit the threshold-energy relation, yielding the absolute energy calibration of the system, as shown in Fig. 4.

The detection efficiency was evaluated using a $10 \mathrm{mCi}{ }^{241} \mathrm{Am} \mathrm{X}$-ray fluorescence source with $\mathrm{Cu}$, $\mathrm{Mo}, \mathrm{Rb}$ and $\mathrm{Ag}$ targets. An absolute calibration was made by detecting the radiation first with an Amptek XR-100 T $\gamma /$ X-ray CZT detector, then with the silicon detectors at appropriate energy thresholds (integrating each energy peak), and taking the ratios of the counting rates. A custom-

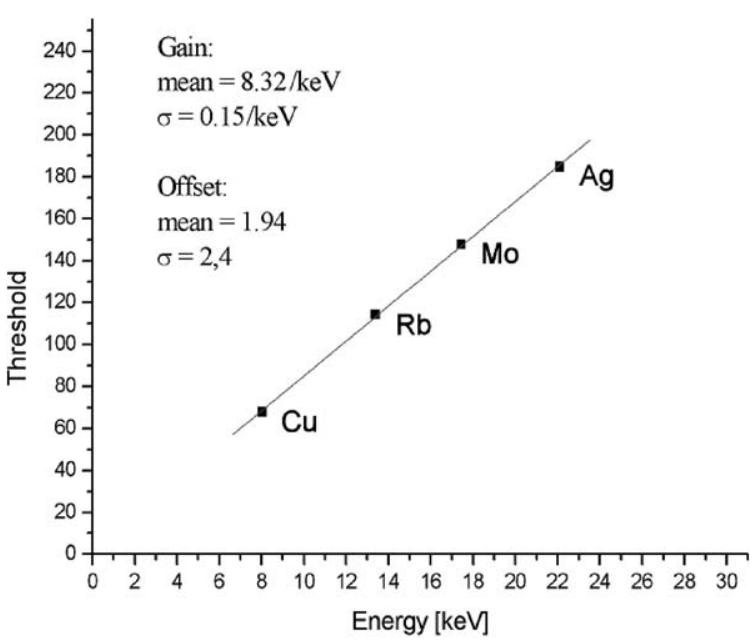

Fig. 4. Energy calibration of the system performed with an ${ }^{241} \mathrm{Am}$ radioactive source and several X-ray fluorescence target materials.

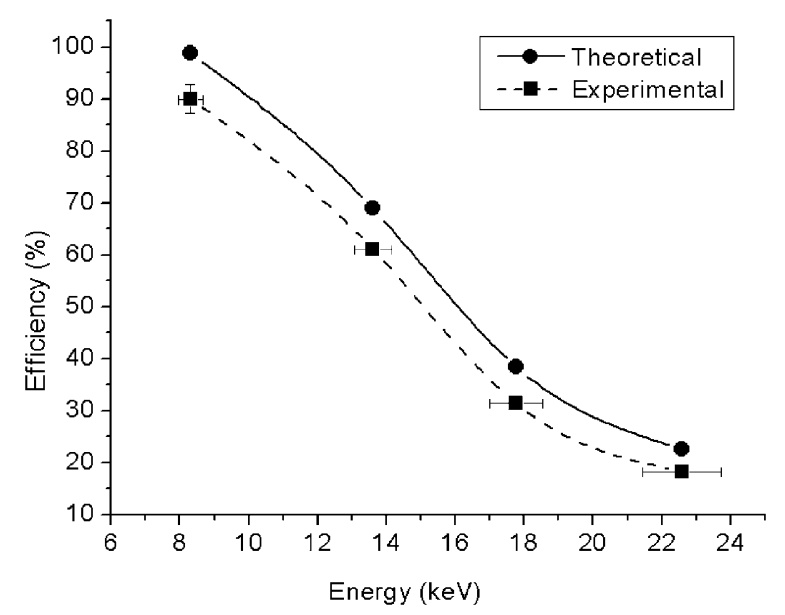

Fig. 5. Detection efficiency of the system as a function of energy: theoretical and experimental points (curves included to guide the eye). The horizontal error bars are due to the averaging of the $\mathrm{K}_{\alpha}$ and $\mathrm{K}_{\beta}$ energies.

made circular collimator $(1.0 \mathrm{~mm}$ diameter on a $15 \mathrm{~mm}$ thick $\mathrm{Pb}$ block) was used in both cases in order to have a well-defined solid angle of emitted radiation from the source hitting the detectors. Absolute normalization was made taking into account the efficiency of the CZT detector specified by the manufacturer. The results are shown in Fig. 5 together with the theoretical values 
computed for a $300 \mu \mathrm{m}$ silicon detector using the NIST X-ray mass attenuation coefficients database [5], each point corresponding to the average energy of $\mathrm{K}_{\alpha}$ and $\mathrm{K}_{\beta} \mathrm{X}$-rays emissions of the target materials, calculated from Ref. [6]. Although the general trend is followed by both sets of points, a systematic difference is observed as the measured efficiency is always $10-20 \%$ lower than the theoretical one. This discrepancy is more significant for lower energies, implying that it may be due to absorptions in the detector cover (made of aluminium foil and black tape, used to block ambient light). Other contributions may come from a detector active thickness smaller than $300 \mu \mathrm{m}$ (though the detector operated above full depletion, the actual thickness of its active area was never directly measured) or a normalization error due to the (3-year old) absolute calibration of the CZT reference detector.

Images were obtained using available low activity $(1-10 \mu \mathrm{Ci})$ radioactive sources. At each collimator slit position, two one-dimensional profiles are obtained at two threshold settings (one below, the other above the value corresponding to the source energy). Direct strip-by-strip subtraction is then performed and the resulting subtracted profile is used as a slice of final image. The results obtained are presented in the next section.

\section{Imaging results}

The first imaging tests have been performed with the single-chip detector board, using a $1 \mu \mathrm{Ci}{ }^{57} \mathrm{Co}$ source. Discriminator levels were set below and above the value corresponding to the $14.4 \mathrm{keV}$ gamma emission of the source, and the scanning step was chosen to be $0.3 \mathrm{~mm}$, the same as the slit window. The source was placed on top of the collimator, aligned with the slit and centred on the detector, as shown in Fig. 2. The acquisition time was several hours due to the low intensity of the source and the reduced geometrical acceptance of the collimator.

The first object to be imaged was a set of $1.0 \mathrm{~mm}$ thick lead masks forming the acronym "LIP". Fig. 6 shows the resulting image, together with a photograph of the test object placed close to a metric ruler; the overall image dimensions are $9.9 \times 6.4 \mathrm{~mm}^{2}$. The image reproduces the main features of the test object, including the imperfections of each single letter. Image contrast goes from 0 (black) to about 80 (white) counts per pixel. Two groups of adjacent dead-channels were present on the detector board, corresponding to the two sets of horizontal black lines.

Repeating the above procedure with the doublechip board, larger objects were imaged. Here examples of high- and low-contrast test objects

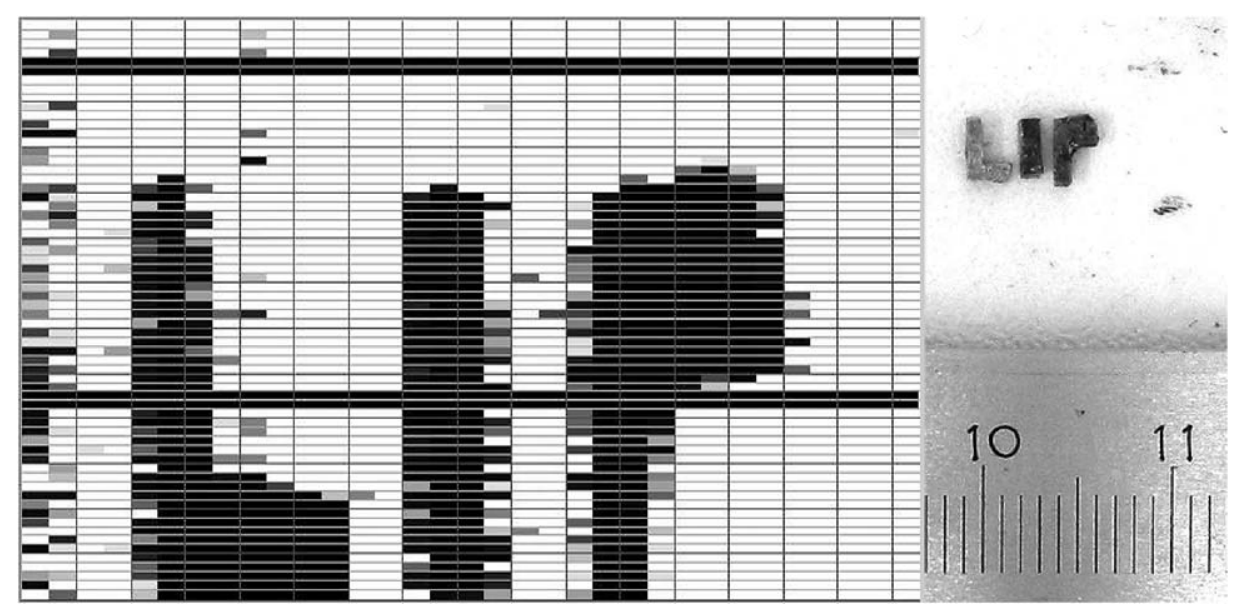

Fig. 6. First image obtained with the single-chip detector board: a set of lead masks forming the word "LIP" (shown on the right against a metric ruler). Image dimensions: $9.9 \times 6.4 \mathrm{~mm}^{2}$. The black horizontal lines correspond to dead channels. 


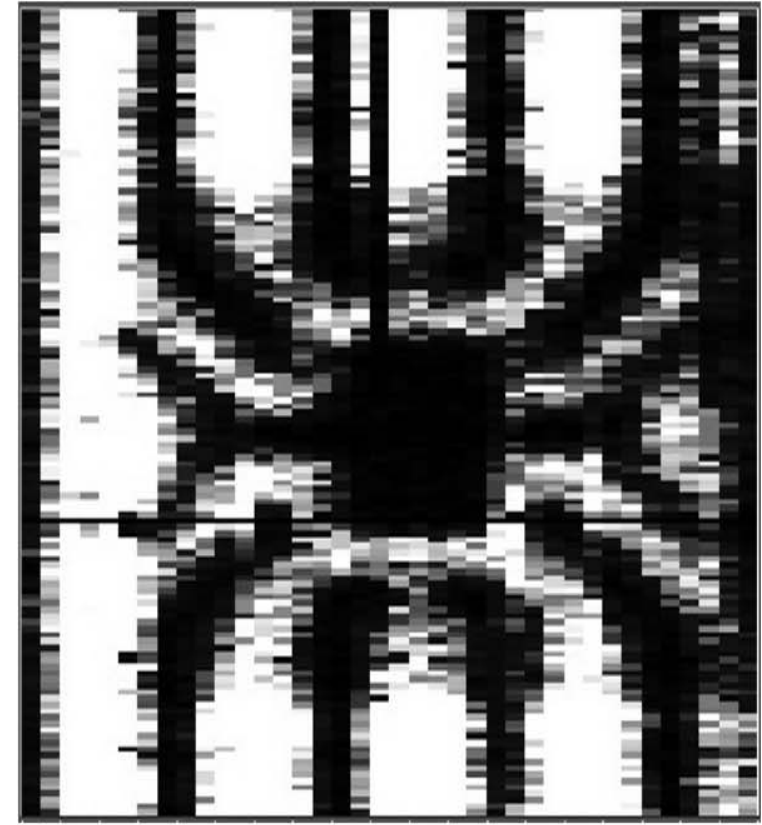

(a)

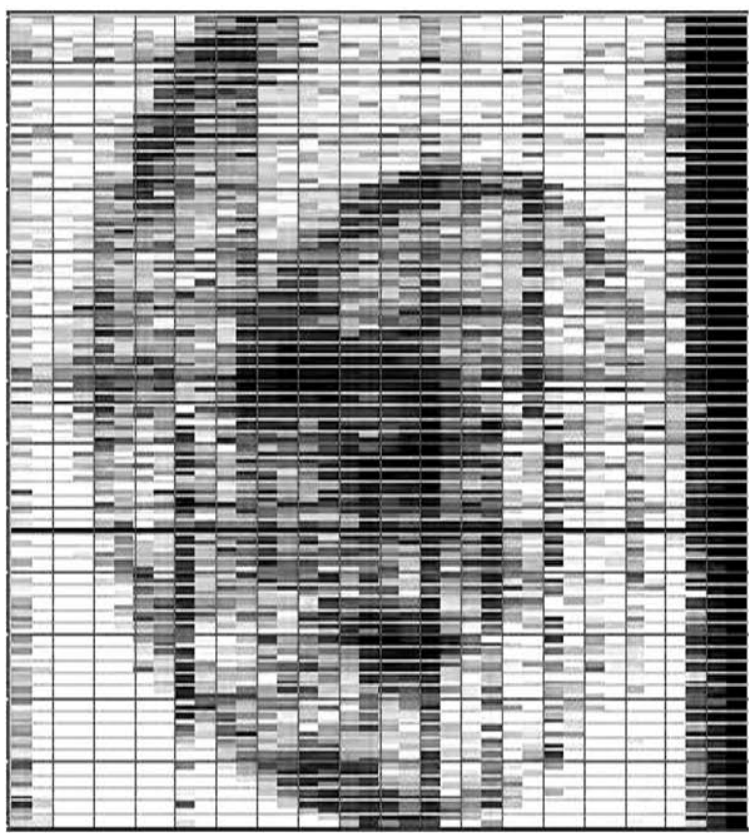

(b)

Fig. 7. Examples of high- and low-contrast images obtained with the double-chip detector board: (a) 8-pin integrated circuit; and (b) snail shell. Image dimensions (both cases): $10.8 \times 12.8 \mathrm{~mm}^{2}$. The black horizontal line corresponds to a dead channel. are presented, namely an 8-pin integrated circuit and a snail shell. Data was taken using a $1.0 \mu \mathrm{Ci}$ ${ }^{109} \mathrm{Cd}$ source, selecting the $22 \mathrm{keV} \mathrm{K} \mathrm{X-rays} \mathrm{of}$ silver. Collimator slit aperture and scanning step were the same as before, namely $0.3 \mathrm{~mm}$. The results are shown in Figs. 7a and b, where the main features of the test objects are seen, more remarkably in the second case; the overall image dimensions are $10.8 \times 12.8 \mathrm{~mm}^{2}$ on both cases. Image contrast goes from 0 to about 100 counts per pixel in the first case, and from 0 to about 400 counts per pixel in the second case.

\section{Conclusions}

A simple system for two-dimensional soft X-ray imaging at normal incidence was implemented, characterised and tested with several radioactive sources and test objects.

The results show good imaging capabilities although the acquisition times are very long due to the use of low intensity radioactive sources. The readout electronics can withstand high counting rates (up to $200 \mathrm{kHz}$ per channel) so that the use of a collimated X-ray tube (not available at the time of this work) would eventually yield better and faster results due to the higher counts per channel and the smaller acquisition time needed to get an image with a good dynamic range.

The main attractions of this system are its simplicity and its modular capability to expand the active area by using larger detectors and adding more readout chips. As future modules for the angiographic detector (see Ref. [3]) will become available, new tests may be performed with larger active areas and (hopefully) an X-ray tube as radiation source, further exploiting and expanding the full potential of this prototype.

\section{Acknowledgements}

P. Rato Mendes, M.C. Abreu, P. Sousa and S. Rodrigues acknowledge financial support in the form of research funds and/or scholarships from Fundação para a Ciência e a Tecnologia, Portugal. 


\section{References}

[1] P. Rato Mendes, Topics on ionising radiation physics and detectors, course at the Fourth Southern European EPS School "Physics in Medicine", Faro, September, 2001.

[2] M. Overdick, Flat X-ray detectors for medical imaging, keynote lecture at the Fourth International Workshop on Radiation Imaging Detectors, Amsterdam, September, 2002, Nucl. Instr. and Meth. A (2003), these proceedings.

[3] L. Ramello, et al., X-ray imaging with a silicon microstrip detector coupled to the RX64 ASIC, oral presentation at the Fourth International Workshop on Radiation Imaging Detectors, Amsterdam, September, 2002, Nucl. Instr. and Meth. A (2003), these proceedings.
[4] W. Dabrowski, et al., RX64 - a fully integrated 64-channel IC for readout of silicon detectors used for X-ray position sensitive measurements, oral presentation to IEEE NSS/ MIC 2001, San Diego, November, 2001.

[5] J.H. Hubbell, S.M. Seltzer, Tables of X-ray mass attenuation coefficients and mass energy-absorption coefficients (version 1.03) (1997) [Online]. Available: http:// physics.nist.gov/xaamdi [2002, October 30]. National Institute of Standards and Technology, Gaithersburg, MD.

[6] A. Thomson, D. Vaughan (Eds.), X-ray Data Booklet (2001) [Online]. Available: http://xdb.lbl.gov [2002, October 30]. Lawrence Berkeley National Laboratory, Berkeley, CA. 